\title{
Asian Americans and Obesity in California: A Protective Effect of Biculturalism
}

\author{
Sophia Wang · Judy Quan · Alka M. Kanaya • \\ Alicia Fernandez
}

Published online: 14 December 2010

(C) Springer Science+Business Media, LLC 2010

\begin{abstract}
Prior studies comparing US-born and foreignborn Asian Americans have shown that birth in the US conveys greater risk of obesity. Our study investigates whether retention of Asian culture might be protective for obesity despite acculturation to US lifestyle. We classified self-identified Asian American respondents of the California Health Interview Survey as traditional, bicultural, and acculturated using nativity and language proficiency in English and Asian language. We then examined the association of acculturation with overweight/obesity (BMI $\geq$ $25 \mathrm{~kg} / \mathrm{m}^{2}$ ) in a multivariate regression model. Acculturated respondents had higher adjusted odds of being overweight/ obese than bicultural respondents (2.13 [1.40-3.23] for men, 3.28 [2.14-5.04] for women), but bicultural respondents had similar odds of being overweight/obese as traditional respondents (.98 [.69-1.41] for men, .72 [.50-1.05] for women). Among the bicultural, second and first generation respondents were equally likely to be overweight/
\end{abstract}

\section{S. Wang}

School of Medicine, University of California,

San Francisco, San Francisco, CA, USA

e-mail: Wang@ucsf.edu

\section{J. Quan · A. Fernandez}

Division of General Internal Medicine, University of California,

San Francisco, San Francisco, CA, USA

e-mail: quanj@medsfgh.ucsf.edu

\section{A. M. Kanaya}

Center for Obesity Assessment, Study, and Treatment,

University of California, San Francisco, San Francisco, CA, USA

e-mail: Alka.Kanaya@ucsf.edu

\section{A. Fernandez ( $\square)$}

San Francisco General Hospital, 1001 Potrero Ave,

Box 1364, San Francisco, CA 94110, USA

e-mail: afernandez@medsfgh.ucsf.edu obese. Biculturalism in Asian Americans as measured by Asian language retention appears protective against obesity. Further research is needed to understand the mechanisms underlying this association.

Keywords Asian Americans - Obesity - Acculturation . Epidemiology

\section{Background and Conceptual Framework}

Asian Americans are less obese than other major US ethnic population groups [1], and lower rates of obesity have been shown to be associated with more favorable health profiles and longer life expectancy [2]. The lower rate of obesity among Asian Americans may be related to the high proportion of immigrants in the Asian American population; studies on obesity in Asian Americans have found that foreign-born Asian Americans are significantly less overweight and obese than US-born Asian Americans, though among foreign-born Asian Americans, increased duration of residence in the US is correlated with increased obesity [3-6]. Studies of Asian Americans by immigrant generational status have observed a trend of increasing obesity with later generations $[1,7]$. Environmental characteristics of the US on a societal level and maladaptive acculturation to US norms of diet and exercise on an individual level are often assumed to explain this phenomenon, but there are no studies linking nativity with behavior change and subsequent obesity in Asian American adults.

Most studies on acculturation and Asian American obesity have relied on nativity, generational status, or length of residence in the US as proxies for acculturation, reflecting in part an underlying linear and progressive view of acculturation, with immigrants' assimilation into the 
White American host society as a final endpoint. Individuals are seen as simultaneously relinquishing the values and behaviors of their heritage culture while adopting the values and behaviors of their host culture in a unidimensional continuum of acculturation. Critics of the concept of acculturation in the medical literature have pointed out that acculturation is often a multidimensional, and nonlinear process whereby individuals adopt new behaviors while retaining other behaviors [8-10], often resulting in bicultural outcomes. Berry's model of acculturation offers up a bidimensional alternative by considering an individual's adoption of host values and behaviors and orientation towards heritage values and behaviors as relatively independent $[9,10]$. This more complex model of acculturation allows individuals who incorporate aspects of multiple cultures to be considered "bicultural," and may be more conceptually informative since it incorporates the effects of gain, loss, and coexistence of heritage and host cultures on health outcomes. Verifying more complex models of acculturation in research examining the relationship between acculturation and obesity in Asian Americans would allow us to identify subpopulations at risk and focus research and interventions on mechanisms of obesity more accurately.

We used data from Asian American respondents from two waves of the California Health Interview Survey (CHIS) to examine the association between acculturation and overweight/obesity (BMI $\geq 25 \mathrm{~kg} / \mathrm{m}^{2}$ ) and to determine if changes in diet and physical activity explain any observed association. We constructed categories of acculturation that allowed for biculturalism by considering an individual's orientation towards US culture and heritage culture relatively independently, as measured by the bidimensional combination of nativity and language ability. Language ability is recognized as a key component of and prerequisite for acculturation and may reveal much about differential processes of acculturation [11, 12]. Among the foreign born, we used fluency in English to indicate the adoption of host culture; among the US-born, we used retention of Asian language ability to distinguish between retention and loss of heritage culture. In addition, we also repeated our analysis of overweight/obesity using the more traditional unidimensional categories of generational status, in order to compare results with our acculturation categories.

\section{Methods}

Sample and Population

We used data from the 2005 and 2007 administrations of the California Health Interview Survey (CHIS), a cross-sectional random-dial telephone survey of the non-institutionalized population of California [13, 14]. CHIS is conducted by the UCLA Center for Health Policy Research, and in 2005, 2007 was administered in English, Spanish, Mandarin, Cantonese, Vietnamese, and Korean. The CHIS sample design was designed to capture information from diverse, small populations and included oversampling of Korean and Vietnamese populations. Accordingly, CHIS used a complex weighting scheme to allow accurate statewide population estimates. These weighted data were used to calculate proportions and throughout the analysis. CHIS includes items on sociodemographics, health status, utilization of health services and health-related behaviors.

We restricted our analysis to adults aged 18 and older who self-reported as Chinese, Vietnamese, Korean, or nonHispanic Whites. Other sizable Asian populations (such as Japanese, Filipino, and South Asian) were excluded because widespread use of English among those ethnic groups did not allow us to use language as a measure of acculturation. We included in our analysis respondents who self-reported their ethnicity as multiple Asian ethnicities so long as one of them was Chinese, Vietnamese, or Korean. Respondents who self-reported as both Asian and non-Hispanic White (Mixed Asian, $\mathrm{N}=93$ ) were included in our sample.

\section{Acculturation Groups}

We constructed three acculturation groups by combining nativity and language proficiency in English and Asian languages. (1) The traditional group included respondents who were foreign-born and reported speaking English "not well" or "not at all." (2) The bicultural group included foreign-born respondents who reported speaking English "well", "very well" or exclusively, and US-born respondents who reported speaking an Asian language at home or answered the CHIS interview in an Asian language. (3) The acculturated group included US-born respondents who spoke English "well”, "very well” or exclusively, answered the CHIS interview in English, and reported not speaking an Asian language at home. In addition, we defined a fourth group comprising US-born, self-reported non-Hispanic Whites who reported speaking English "well," "very well," or exclusively, in order to anchor rates of overweight/obesity in Asian Americans within the broader Californian population.

Measures

All variables were based on respondents' self-reports. We used self-reported weight and height to calculate respondents' body mass index $\left(\mathrm{BMI}=\right.$ weight $[\mathrm{kg}] /$ height $[\mathrm{m}]^{2}$ ) and defined our main outcome of overweight/obesity as 
BMI $\geq 25 \mathrm{~kg} / \mathrm{m}^{2}$, which is considered overweight by the WHO international standard [15] and obese for Asian populations [16]. Possible confounding variables included age; ethnicity (self-reported Chinese, Korean, Vietnamese, and Mixed); the socioeconomic factors of educational attainment and income (represented in multiples of $100 \%$ federal poverty level, capped at 24 or $2,400 \%$ federal poverty level); covariates of marital status and health care use (defined as visiting a doctor in the past 12 months); and finally, the individual risk behavior of tobacco use.

Diet and exercise behavior may mediate the relationship between acculturation status and BMI. CHIS includes questions on frequencies of fruit, vegetable, French fry, and soda consumption based on 30-day recall, as well as frequencies and duration of moderate and vigorous physical activities based on 7-day recall. Consumption of fruits and vegetables were added to form one mediator indicating number of times fruits and vegetables were consumed per week. Consumption of fries and soda were similarly added to form another mediator indicating number of times fries and soda were consumed per week. Physical activity variables included whether the respondent engaged in vigorous leisure-time physical activity at least 3 days/week and $20 \mathrm{~min} /$ day, and whether the respondent had a sedentary lifestyle (did not engage in vigorous leisure-time activity 3 days/week for at least $20 \mathrm{~min} /$ day or in moderate leisuretime activity 5 day/week for at least $30 \mathrm{~min} /$ day) [17].

\section{Data Analysis}

As the relationship between acculturation and obesity has been observed to vary by gender [18-21], and since testing for interaction by gender in our sample was significant $(P=.0001)$, all analyses were stratified by gender. We compared the distribution of possible confounding and mediating variables between the acculturation categories using a Pearson-type chi-square test for categorical variables and the Wald chi-square test for interval variables. Age-adjusted percentages of overweight/obesity in each acculturation category were calculated. Percentage overweight/obese of the non-Hispanic White group was also calculated as a reference, age adjusting across all Asians and non-Hispanic Whites. We used logistic regression models to examine the independent association of acculturation category and overweight/obesity, using the Traditional category as a reference. Models were created by sequentially adding confounders followed by mediators to the model.

Because the sample was composed of several ethnic subgroups (Chinese, Korean, Vietnamese, and Mixed) we repeated our analysis stratified by ethnic subgroup. The analysis was also repeated excluding respondents who classified themselves as both Asian and White. Finally, to compare our results with those of prior studies that use generational status as a marker for acculturation, we also regrouped our respondents by generational status and repeated our logistic regression modeling. Respondents were considered first generation if they reported being foreignborn, second generation if at least one parent was foreignborn, and third generation or higher if neither parent was foreign-born.

All statistical analysis was performed using SUDAAN 10.0 to accommodate population weights.

The present study was approved by the UCSF Committee on Human Research.

\section{Results}

Population Characteristics and Behaviors

The combined 2005 and 2007 CHIS data yielded a total of 63,030 eligible respondents, representing approximately 27.3 million non-institutionalized Californian adults. Of these, 5,074 were self-reported Chinese, Vietnamese, or Koreans; the remaining were English-speaking US-born non-Hispanic Whites. Distribution of ethnicities differed among the three acculturation categories; the acculturated group had a higher proportion of Chinese respondents (Tables 1, 2).

Characteristics of the three Asian American acculturation groups are presented for men in Table 1 and women in Table 2. Differences in population characteristics between acculturation categories were most striking in the SES variables of education and income: bicultural and acculturated respondents had higher levels of education and income compared to the traditional respondents. Differences between acculturation groups in the health behavior of smoking were also striking. For women, the acculturated group had a higher percentage of smokers, whereas in men the traditional group had a higher percentage of smokers. Differences between other health behaviors were less prominent, though for both sexes, there was a graded response in fries and soda consumption, with the bicultural group being intermediate between the traditional and acculturated groups. Also for both sexes, acculturated respondents reported more vigorous leisure-time physical activity than traditional respondents.

Tables 1 and 2 also display the distribution of the different immigrant generations in each acculturation category. The bicultural group largely comprises first and second generation respondents (82.6 and $17.2 \%$ for men, 84.2 and $15.7 \%$ for women). The acculturated group comprises second and higher generations (62.8 and $37.3 \%$ for men, 75.3 and $24.7 \%$ for women). In addition, almost all third or higher generation respondents were categorized as acculturated. 
Table 1 Demographic characteristics and health behaviors of Californian Asian American men

\begin{tabular}{|c|c|c|c|c|c|c|c|}
\hline & \multicolumn{2}{|c|}{$\begin{array}{l}\text { Traditional } \\
(\mathrm{N}=749)^{\mathrm{a}}\end{array}$} & \multicolumn{2}{|c|}{$\begin{array}{l}\text { Bicultural } \\
(\mathrm{N}=1,164)^{\mathrm{b}}\end{array}$} & \multicolumn{2}{|c|}{$\begin{array}{l}\text { Acculturated } \\
(\mathrm{N}=253)^{\mathrm{c}}\end{array}$} & \multirow[t]{2}{*}{$P$ value } \\
\hline & $\%$ & SE & $\%$ & SE & $\%$ & SE & \\
\hline Age (Mean/SE) & 53.0 & 0.92 & 38.7 & 0.59 & 37.4 & 1.22 & $<.0001$ \\
\hline \multicolumn{8}{|l|}{ Ethnicity $^{\mathrm{d}}$} \\
\hline Chinese & 47.71 & 2.48 & 58.47 & 2.02 & 80.39 & 3.31 & $<.0001$ \\
\hline Korean & 19.76 & 1.58 & 19.20 & 1.33 & 16.55 & 3.35 & .6861 \\
\hline Vietnamese & 35.52 & 2.38 & 24.93 & 1.75 & 5.44 & 1.52 & $<.0001$ \\
\hline Educational attainment & & & & & & & $<.0001$ \\
\hline High school and below & 61.24 & 2.84 & 21.88 & 1.72 & 24.41 & 4.01 & \\
\hline Some college, vocational school, AA or AS & 11.19 & 1.85 & 19.25 & 1.91 & 20.53 & 3.36 & \\
\hline College and beyond & 27.58 & 2.52 & 58.88 & 2.01 & 55.06 & 4.02 & \\
\hline Poverty level $\times 100 \%($ Mean/SE) & 2.42 & 0.12 & 5.37 & 0.185 & 6.27 & 0.35 & $<.0001$ \\
\hline Marital status & & & & & & & $<.0001$ \\
\hline Married & 81.21 & 2.59 & 56.89 & 2.52 & 45.56 & 4.01 & \\
\hline Other/widowed/separated/divorced/living with partner & 8.46 & 1.67 & 5.29 & 1.23 & 11.44 & 2.18 & \\
\hline Never married & 10.34 & 2.35 & 37.82 & 2.43 & 43.01 & 4.14 & \\
\hline Visited a doctor in the last 12 months & 68.63 & 2.35 & 67.66 & 2.23 & 75.75 & 3.71 & .1967 \\
\hline Smoking & & & & & & & $<.0001$ \\
\hline Current smoker & 31.44 & 2.60 & 17.46 & 1.77 & 12.67 & 2.76 & \\
\hline Quit smoking & 31.98 & 2.23 & 18.62 & 1.68 & 16.19 & 2.86 & \\
\hline Never regularly smoked & 36.59 & 2.48 & 63.92 & 2.17 & 71.14 & 3.75 & \\
\hline \multicolumn{8}{|l|}{ Vigorous leisure-time physical activity } \\
\hline At least 3 days/week and $20 \mathrm{~min} /$ day & 7.18 & 1.23 & 16.97 & 1.59 & 18.10 & 3.23 & $<.0001$ \\
\hline \multicolumn{8}{|l|}{ Regular physical activity } \\
\hline No physical activity (sedentary) & 19.91 & 2.58 & 13.52 & 1.79 & 10.22 & 4.36 & .0732 \\
\hline \# of times consumed fruits and vegetables in the last 7 days & 13.10 & 0.40 & 12.96 & 0.35 & 11.65 & 0.76 & .2320 \\
\hline \# of times consumed fries and soda in the last 7 days & 1.99 & 0.24 & 2.87 & 0.17 & 3.85 & 0.38 & .0001 \\
\hline Generation & & & & & & & $<.0001$ \\
\hline First generation & 100 & 0.00 & 82.59 & 2.02 & 0.00 & 0.00 & \\
\hline Second generation & 0.00 & 0.00 & 17.21 & 2.00 & 62.75 & 4.16 & \\
\hline Third generation or higher & 0.00 & 0.00 & 0.20 & 0.16 & 37.25 & 4.16 & \\
\hline BMI (Mean/SE)* & 24.31 & 0.29 & 23.86 & 0.16 & 25.08 & 0.26 & $<.0001$ \\
\hline Overweight or obese (BMI $\geq 25)^{*}$ & 33.74 & 3.04 & 32.95 & 1.96 & 50.02 & 4.43 & .0029 \\
\hline
\end{tabular}

Data from respondents to California Health Interview Survey 2005, 2007

*All values are age adjusted

a Traditional: foreign-born respondents who reported speaking English "not well" or "not at all"

b Bicultural: Foreign-born respondents who reported speaking English "well”, "very well" or exclusively, and US-born respondents who reported speaking an Asian language at home or answered the CHIS interview in an Asian language

c Acculturated: US-born respondents who spoke English "well", "very well" or exclusively, answered the CHIS interview in English, and reported not speaking an Asian language at home

${ }^{d}$ Ethnicity column percentages may not sum to $100 \%$ because some respondents self-identified as more than one Asian ethnicity

Differences in BMI by Acculturation and Generational Status

Figure 1 displays the percent overweight/obese for each acculturation category and generation. Among women, bicultural respondents were less likely to be overweight/ obese than acculturated respondents (12.5 vs. 34.0\%, $P<.0001)$ and traditional respondents $(12.5$ vs. $18.6 \%$, $P<.0001)$. Bicultural men also had a significantly lower percentage of overweight/obese respondents than acculturated men (33.0 vs. $50.0 \%, P=.0008$ ), but showed no significant difference in percentage of overweight/obese 
Table 2 Demographic characteristics and health behaviors of Californian Asian American women

\begin{tabular}{|c|c|c|c|c|c|c|c|}
\hline & \multicolumn{2}{|c|}{$\begin{array}{l}\text { Traditional } \\
(\mathrm{N}=1,200)^{\mathrm{a}}\end{array}$} & \multicolumn{2}{|c|}{$\begin{array}{l}\text { Bicultural } \\
(\mathrm{N}=1,368)^{\mathrm{b}}\end{array}$} & \multicolumn{2}{|c|}{$\begin{array}{l}\text { Acculturated } \\
(\mathrm{N}=340)^{\mathrm{c}}\end{array}$} & \multirow[t]{2}{*}{$P$ value } \\
\hline & $\%$ & SE & $\%$ & SE & $\%$ & SE & \\
\hline Age $($ Mean/SE) & 52.0 & 0.64 & 38.0 & 0.62 & 37.2 & 1.27 & $<.0001$ \\
\hline \multicolumn{8}{|l|}{ Ethnicity $^{\mathrm{d}}$} \\
\hline Chinese & 47.57 & 1.70 & 61.47 & 2.02 & 76.51 & 3.98 & $<.0001$ \\
\hline Korean & 25.59 & 1.41 & 17.08 & 1.29 & 14.38 & 3.61 & .0004 \\
\hline Vietnamese & 28.75 & 1.63 & 23.24 & 1.82 & 9.17 & 2.74 & $<.0001$ \\
\hline Educational attainment & & & & & & & $<.0001$ \\
\hline High school and below & 68.20 & 1.67 & 21.91 & 2.18 & 23.05 & 3.69 & \\
\hline Some college, vocational school, AA or AS & 11.29 & 1.27 & 18.39 & 1.95 & 21.8 & 3.84 & \\
\hline College and beyond & 20.51 & 1.50 & 59.71 & 2.10 & 55.15 & 4.55 & \\
\hline Poverty level $\times 100 \%($ Mean/SE) & 2.35 & 0.10 & 5.178 & 0.17 & 6.44 & 0.38 & $<.0001$ \\
\hline Marital status & & & & & & & $<.0001$ \\
\hline Married & 72.62 & 2.08 & 55.73 & 2.28 & 43.48 & 4.01 & \\
\hline Other/widowed/separated/divorced/Living with partner & 20.28 & 1.61 & 9.80 & 1.17 & 17.77 & 3.45 & \\
\hline Never married & 7.10 & 1.58 & 34.48 & 2.29 & 38.75 & 4.35 & \\
\hline Visited a doctor in the last 12 months & 73.18 & 1.89 & 82.77 & 1.90 & 89.96 & 1.90 & $<.0001$ \\
\hline Smoking & & & & & & & $<.0001$ \\
\hline Current smoker & 3.14 & 0.95 & 4.67 & 1.07 & 8.15 & 2.38 & \\
\hline Quit smoking & 2.40 & 0.53 & 7.48 & 1.20 & 13.33 & 2.90 & \\
\hline Never regularly smoked & 94.46 & 1.06 & 87.85 & 1.55 & 78.52 & 3.39 & \\
\hline \multicolumn{8}{|l|}{ Vigorous leisure-time physical activity } \\
\hline At least 3 days/week and $20 \mathrm{~min} /$ day & 5.35 & 0.95 & 7.80 & 1.02 & 13.99 & 2.86 & .0177 \\
\hline \multicolumn{8}{|l|}{ Regular physical activity } \\
\hline No physical activity (sedentary) & 21.42 & 1.98 & 13.43 & 1.37 & 8.95 & 2.38 & .0004 \\
\hline \# of times consumed fruits and vegetables in the last 7 days & 14.81 & 0.32 & 15.42 & 0.39 & 16.79 & 0.84 & .0681 \\
\hline \# of times consumed fries and soda in the last 7 days & 0.805 & 0.11 & 1.38 & 0.12 & 2.17 & 0.28 & $<.0001$ \\
\hline Generation & & & & & & & $<.0001$ \\
\hline First generation & 100 & 0.00 & 84.21 & 2.16 & 0.00 & 0.00 & \\
\hline Second generation & 0.00 & 0.00 & 15.70 & 2.16 & 75.32 & 3.11 & \\
\hline Third generation or higher & 0.00 & 0.00 & 0.08 & 0.06 & 24.68 & 3.11 & \\
\hline BMI (Mean/SE)* & 22.61 & 0.19 & 21.99 & 0.14 & 23.75 & 0.36 & $<.0001$ \\
\hline Overweight or obese (BMI $\geq 25)^{*}$ & 18.54 & 1.51 & 12.52 & 1.29 & 33.95 & 3.86 & $<.0001$ \\
\hline
\end{tabular}

Data from respondents to California Health Interview Survey 2005, 2007

*All values are age adjusted

a Traditional: foreign-born respondents who reported speaking English "not well" or "not at all"

b Bicultural: Foreign-born respondents who reported speaking English "well", "very well" or exclusively, and US-born respondents who reported speaking an Asian language at home or answered the CHIS interview in an Asian language

c Acculturated: US-born respondents who spoke English "well”, "very well” or exclusively, answered the CHIS interview in English, and reported not speaking an Asian language at home

${ }^{\mathrm{d}}$ Ethnicity column percentages may not sum to $100 \%$ because some respondents self-identified as more than one Asian ethnicity

compared to traditional men (33.0 vs. $33.7 \%$ ). Men had higher percentages of being overweight/obese than women in every acculturation category. Non-Hispanic White respondents had a higher percentage of overweight/obese (65.2\% for men, $46.3 \%$ for women) than the most acculturated group of Asians for both men and women. When the analysis was repeated for individual ethnic groups, the overall pattern of acculturated respondents having higher percentages of overweight/ obesity than traditional and bicultural respondents was similar for women in all ethnic subgroups and for men who were Chinese or Mixed Asian. Analysis of Korean 
and Vietnamese men was hampered by the sample size of the acculturated group.

Table 3 presents the odds of being overweight/obese for bicultural and acculturated Asians Americans compared with traditional Asian Americans. Each row displays the odds ratio after the successive addition of groups of confounding or mediating variables to the model. The acculturated group had higher odds of being overweight/obese than the traditional group at each successive variable addition, with a final adjusted odds ratio of 2.11 [1.25-3.55] for men and 2.38 [1.41-4.01] for women when all variables were in the model. The odds ratio did not change substantially with the stepwise addition of sociodemographic or other covariates. Furthermore, addition of diet and exercise mediators to the model reduced the odds ratio only minimally. Direct comparison between the bicultural and the acculturated group using the bicultural group as the reference further confirmed the protectiveness of biculturalism, with the acculturated group having higher odds of being overweight/obese (2.13 [1.40-3.23] in men, 3.28 [2.14-5.04] in women), with all variables in the model.

Figure 1 displays age adjusted rate of overweight/ obesity by generational status as well as by our acculturation categories. For both sexes, the bicultural respondents were less likely than second generation respondents to be overweight/obese ( 33.0 vs. $39.1 \%$ for men) with particularly large differences for women (12.5 vs. $22.0 \%)$. In order to confirm that the association of less overweight/ obesity with biculturalism was not driven by the large percentage of first generation respondents categorized as

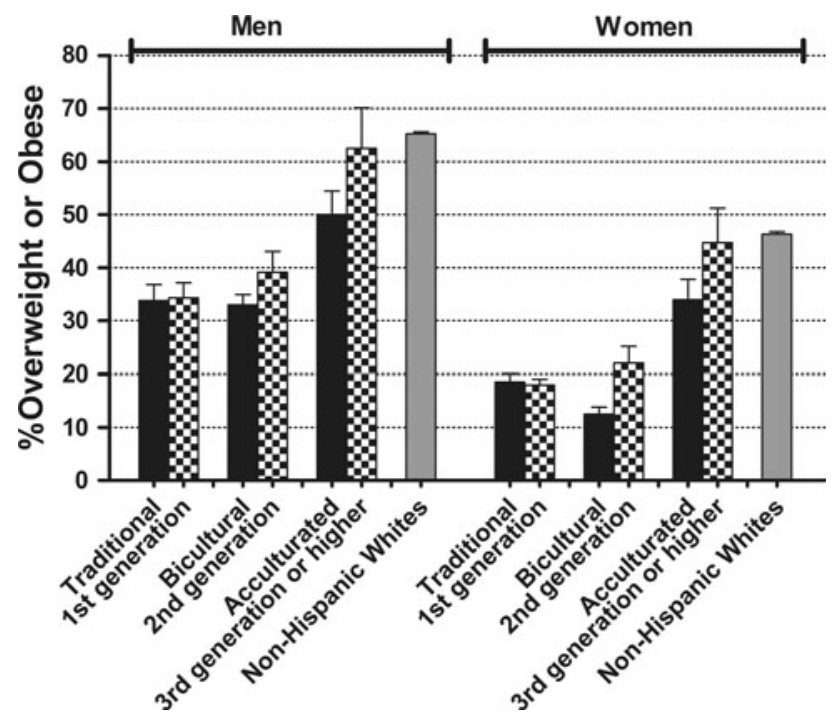

Fig. 1 Age-adjusted percent overweight or obese by Asian American acculturation category and generational status. Age-adjusted percentage of respondents in each category who had BMI $\geq 25$ are plotted as bars, with error bars representing standard error of mean. Solid bars represent respondents grouped by acculturation category; checkered bars represent respondents grouped by increasing generational status bicultural, we further stratified the bicultural group into first and second generation respondents. We found little difference in age-adjusted percentage of overweight/obesity between first and second generation bicultural respondents ( 33.6 vs. $29.7 \%$ for men, 12.4 vs. $13.5 \%$ for women) and repeated multivariable modeling showed no differences in the effects of confounding or mediating variables. To test the hypothesis that second generation respondents were more overweight/obese than bicultural respondents due to the presence of acculturated respondents in the second generation group, we stratified second generation respondents by acculturation category. We found that among the second generation respondents, acculturated respondents were more likely to be obese than bicultural respondents ( 41.3 vs. $29.3 \%, P=.1407, \mathrm{~N}=306$ for men; 25.1 vs. $10.3 \%, P=.0015, \mathrm{~N}=377$ for women).

\section{Discussion}

Our study of a population-based sample of Asian Americans in California found that acculturation categories based on nativity and language were independently associated with overweight/obesity and that bicultural Asian Americans, despite an education and income profile similar to that of acculturated Asian Americans, had rates of overweight/obesity similar to those found among traditional Asians. While our study is consistent with prior studies indicating that US-born Asian Americans are more likely to be overweight/obese than foreign-born Asian Americans, our results add to this literature by suggesting that cultural orientation, rather than simply birth and life in the US environment, may influence obesity rates.

Analysis of Asian Americans by both acculturation status and generational status each revealed unique insights into mechanisms contributing to overweight/obesity. We found that the bicultural group had a low percentage of overweight/obesity compared to the acculturated group, which suggests that loss of heritage culture rather than gain of host culture is a key factor in development of overweight/obesity, particularly for women. Furthermore, we found that third or higher generation respondents (almost all of whom were acculturated) had higher percentages of overweight/obesity than acculturated respondents overall. This suggests a synergistic influence between increasing generational status and the loss of heritage orientation on overweight/obesity, perhaps due to a set of broader cultural changes in behaviors that are associated with generational status.

Some of the cultural changes that may lead to overweight/obesity are manifest in respondents' reports of physical activity and consumption of fries and soda. Unhealthy consumption of fries and soda increased with acculturation, 
Table 3 Odds ratios for overweight/obesity $\left(\mathrm{BMI} \geq 25 \mathrm{~kg} / \mathrm{m}^{2}\right.$ ) by Asian American acculturation category

\begin{tabular}{|c|c|c|c|c|c|c|c|c|}
\hline \multirow[t]{3}{*}{ Adjusting for... } & \multicolumn{4}{|c|}{ Men } & \multicolumn{4}{|c|}{ Women } \\
\hline & \multicolumn{2}{|c|}{$\begin{array}{l}\text { Bicultural } \\
(\mathrm{N}=1,164)^{\mathrm{a}}\end{array}$} & \multicolumn{2}{|c|}{$\begin{array}{l}\text { Acculturated } \\
(\mathrm{N}=253)^{\mathrm{b}}\end{array}$} & \multicolumn{2}{|c|}{$\begin{array}{l}\text { Bicultural } \\
(\mathrm{N}=1,368)^{\mathrm{a}}\end{array}$} & \multicolumn{2}{|c|}{$\begin{array}{l}\text { Acculturated } \\
(\mathrm{N}=340)^{\mathrm{b}}\end{array}$} \\
\hline & OR & $95 \% \mathrm{CI}$ & OR & $95 \% \mathrm{CI}$ & OR & $95 \% \mathrm{CI}$ & OR & $95 \% \mathrm{CI}$ \\
\hline +Age, Ethnicity & .97 & $.70-1.34$ & 2.08 & $1.30-3.34$ & .62 & $.46-.84$ & 2.18 & $1.45-3.26$ \\
\hline +Educational attainment, Poverty level & .94 & $.66-1.32$ & 2.00 & $1.22-3.28$ & .76 & $.51-1.13$ & 2.66 & $1.63-4.34$ \\
\hline +Marital status, Visited a doctor in the last 12 months & .99 & $.70-1.42$ & 2.12 & $1.25-3.60$ & .77 & $.52-1.14$ & 2.69 & $1.60-4.52$ \\
\hline +Smoking & .98 & $.69-1.40$ & 2.10 & $1.23-3.58$ & .73 & $.51-1.06$ & 2.52 & $1.50-4.25$ \\
\hline $\begin{array}{l}\text { +Vigorous leisure-time physical activity, } \\
\text { Regular physical activity (not sedentary) }\end{array}$ & .99 & $.70-1.42$ & 2.17 & $1.28-3.66$ & .74 & $.51-1.06$ & 2.53 & $1.49-4.28$ \\
\hline $\begin{array}{l}+ \text { Fruit and vegetable consumption, } \\
\text { Fries and soda consumption }\end{array}$ & .98 & $.69-1.41$ & 2.11 & $1.25-3.55$ & .72 & $.50-1.05$ & 2.38 & $1.41-4.01$ \\
\hline
\end{tabular}

Reference group: Traditional: foreign-born respondents who reported speaking English "not well" or "not at all"

a Bicultural: Foreign-born respondents who reported speaking English "well”, "very well" or exclusively, and US-born respondents who reported speaking an Asian language at home or answered the CHIS interview in an Asian language

b Acculturated: US-born respondents who spoke English "well”, "very well" or exclusively, answered the CHIS interview in English, and reported not speaking an Asian language at home

consistent with US norms. Interestingly, physical activity increased with acculturation as well, which is consistent with a study that reported that US-born Asians have increased leisure-time physical activity compared to foreign born Asians Americans [22]. In our study, neither diet nor exercise patterns strongly explained the association between acculturation and overweight/obesity. This surprising result may be because the measures of diet and exercise in this dataset did not truly capture more subtle differences in diet and exercise behavior, and consequently were weak proxies for real-life behavior. Thus, the effects of cultural change on obesity are likely mediated by a broader set of behaviors or factors not captured by our study.

Also left to explain is the suggestion that acculturation may have higher impact on women than on men with respect to overweight/obesity, or that biculturalism may be more protective in women than in men. Gendered cultural norms and values could certainly play a role in the differential effect of acculturation on each gender. Further exploration of the impact of gender on acculturation may be an avenue of additional research.

Other studies have found a protective role of biculturalism in child and adolescent mental health and social functioning. Recent studies of acculturation among children and adolescents of first generation immigrants of many ethnicities, including Hispanic and Asian, link fluent bilingualism in English and heritage language with higher academic achievement, higher educational and occupational ambitions, higher self esteem and decreased depression [11]. Involvement of Latino teens in parental culture of origin was found to be positively related to self esteem [23]. Fluent bilingualism in children of immigrants is associated with "selective acculturation [, which] takes place when the learning process of both generations is embedded in a co-ethnic community of sufficient size and institutional diversity to slow down the cultural shift and promote partial retention of the parents' home language and norms" [11]. Our study appears to extend the benefits of biculturalism and selective acculturation beyond children and adolescents and to the realm of physical health, at least among Asian Americans. This may differ in other ethnic groups. Studies of Latino immigrants have noted that although obesity tends to increase with increasing generations and duration of residence in the US, among Latina women, English preference [18, 24] or higher levels of assimilation [25] is actually associated with lower obesity, suggesting a protective effect conferred by acquisition of host culture. Differences in obesity patterns between ethnic groups may be partly explained by segmented assimilation to diverse subcultures and communities within the US.

Our study has several limitations. The study was conducted on a California population sample and may not generalize to Asian Americans elsewhere. We relied on respondents' self report of weight and height and health behaviors, which is subject to recall error and bias. Social desirability leading to underreporting of weight may differ by acculturation status, potentially leading to conservative differences in obesity rates. We had no direct measure of acculturation and included language ability as a proxy, as is commonly done. As a result of using proxy measures for acculturation, some misclassification of acculturation status remains a possibility. We also had no measure of parity, 
which contributes to obesity in women. Sample size limitations did not allow for complete analysis of individual Asian ethnic groups, and our results may not extend to other Asian groups with different cultural and religious practices such as Japanese, Filipino, or South Asian. Finally, a cross-sectional study cannot prove a causal link between acculturation and development of obesity, although reverse causation is unlikely in this case.

\section{Conclusions}

In conclusion, we found that biculturalism among Asian Americans, as measured by retention of Asian language, is protective against overweight/obesity as compared to being acculturated without retention of Asian language. The mechanism of this association remains to be elucidated; further research is needed determine why retention of heritage culture is protective and how protective behaviors can be extended despite increasing generational status. As the population of Asian Americans continues to rise, clinicians will see an increasing number of both recent immigrants and acculturating generations; our study pinpoints those who have lost their heritage language and culture as particularly at risk for becoming overweight/ obese. Promoting healthy behaviors associated with the heritage culture among English-speaking Asian Americans may enhance the protectiveness of selective acculturation and delay or reduce the maladaptive effects of US acculturation on obesity.

Acknowledgments Alicia Fernandez, MD efforts were supported in part by an award from the Arnold P. Gold Foundation. Sophia Wang efforts were supported by UCSF Dean's Summer Research Fellowship.

\section{References}

1. Bates LM, Acevedo-Garcia D, Alegría M, Krieger N. Immigration and generational trends in body mass index and obesity in the United States: results of the National Latino and Asian American Survey, 2002-2003. Am J Public Health. 2008;98(1):70-7.

2. Singh GK, Siahpush M. Ethnic-immigrant differentials in health behaviors, morbidity, and cause-specific mortality in the United States: an analysis of two national data bases. Hum Biol. 2002; 74(1):83-109.

3. Kaushal N. Adversities of acculturation? Prevalence of obesity among immigrants. Health Econ. 2009;18(3):291-303.

4. Lauderdale DS, Rathouz PJ. Body mass index in a US national sample of Asian Americans: effects of nativity, years since immigration and socioeconomic status. Int J Obes Relat Metab Disord. 2000;24(9):1188-94.

5. Goel MS, McCarthy EP, Phillips RS, Wee CC. Obesity among US immigrant subgroups by duration of residence. JAMA. 2004;292(23):2860.
6. Sanchez-Vaznaugh EV, Kawachi I, Subramanian SV, Sanchez BN, Acevedo-Garcia D. Do socioeconomic gradients in body mass index vary by race/ethnicity, gender, and birthplace? Am J Epidemiol. 2009;169(9):1102.

7. Singh GK, Kogan MD, Yu SM. Disparities in obesity and overweight prevalence among us immigrant children and adolescents by generational status. J Community Health. 2009;34(4):271-81.

8. Abraido-Lanza AF, Armbrister AN, Florez KR, Aguirre AN. Toward a theory-driven model of acculturation in public health research. Am J Public Health. 2006;96(8):1342.

9. Berry JW. Acculturation and adaptation in a new society. Int Migration. 1992;30:69-85.

10. Berry JW. Immigration, acculturation, and adaptation. Appl Psychol. 1997;46(1):5-34.

11. Portes A, Rumbat RG. Legacies: the story of the immigrant second generation [Internet]. Berkeley: University of California Press Russell Sage Foundation; 2001. Available from: http://ark. cdlib.org/ark:/13030/kt629020v5/.

12. Kang S. Measurement of acculturation, scale formats, and language competence: their implications for adjustment. J Cross Cultural Psychol. 2006;37(6):669-93.

13. California Health Interview Survey. CHIS 2005 adult public use file [internet]. Los Angeles, CA: UCLA Center for Health Policy Research; 2008. Available from: http://www.chis.ucla.edu/main/ PUF/download_2005.asp.

14. California Health Interview Survey. CHIS 2007 adult public use file [internet]. Los Angeles, CA: UCLA Center for Health Policy Research; 2008. Available from: http://www.chis.ucla.edu/main/ PUF/download_2007.asp.

15. Obesity: preventing and managing the global epidemic. Report of a WHO consultation. World Health Organ Tech Rep Ser 2000; 894:i-xii (1-253).

16. The Asia-Pacific Perspective: Redefining obesity and its treatment. World Health Organization; 2000.

17. California Health Interview Survey. Constructed variables CHIS 2005 adult survey [internet]. Los Angeles, CA: UCLA Center for Health Policy Research; 2009. Available from: http://www.chis. ucla.edu/main/PUF/download_2005.asp\#constructs.

18. Sundquist J, Winkleby M. Country of birth, acculturation status and abdominal obesity in a national sample of Mexican-American women and men. Int J Epidemiol. 2000;29(3):470-7.

19. Ahn MK, Juon HS, Gittelsohn J. Association of race/ethnicity, socioeconomic status, acculturation, and environmental factors with risk of overweight among adolescents in California, 2003. Prev Chronic Dis. 2008;5(3):A75.

20. Antecol H, Bedard K. Unhealthy assimilation: why do immigrants converge to American health status levels? Demography 2006;43(2):337-360.

21. Oza-Frank R, Cunningham SA. The weight of US residence among immigrants: a systematic review [internet]. Obes Rev 2009. Available from: http://www.ncbi.nlm.nih.gov/pubmed/195 38440.

22. Kandula NR, Lauderdale DS. Leisure time, non-leisure time, and occupational physical activity in Asian Americans. Ann Epidemiol. 2005;15(4):257-65.

23. Smokowski P, Buchanan RL, Bacallao ML. Acculturation and adjustment in Latino adolescents: how cultural risk factors and assets influence multiple domains of adolescent mental health. J Prim Prev. 2009;30(3-4):371-93.

24. Khan LK, Sobal J, Martorell R. Acculturation, socioeconomic status, and obesity in Mexican Americans, Cuban Americans, and Puerto Ricans. Int J Obes Relat Metab Disord. 1997;21(2):91-6.

25. Hazuda HP, Mitchell BD, Haffner SM, Stern MP. Obesity in Mexican American subgroups: findings from the San Antonio Heart study. Am J Clin Nutr. 1991;53(6 Suppl):1529S-34S. 